\title{
Volumetric
}

\section{representations could revolutionise 3D printing}

The world of manufacturing is facing a massive game change. For half a century, subtractive manufacturing (SM) has reigned king. In SM, a cutter removes material from a homogeneous block unti Additive manufacturing is a Additive manufacturing is a around the corner - and with it, the opportunity to design in a more flexible way. However, current geometric modelling software does not allow manufacturers to effectively design, analyse, optimise and and/or porous heterogeneous and/or porous objects usin additive manufacturing. in the Computer Science Department at Technion in Israel, has a new approach.

Ow much do you value the 'hand- Using AM, manufacturers have the crafted' over the 'machine-built'? ability to create objects which were Ter waps not as much as you think. would wager that amongst the most Important things you own - things which were designed purely for a practical their be, or things which you admire for were designed by a computer.

Your watch, car, computer and smart phone will all contain parts which were digitally designed and then milled, lathed, cut, moulded and machined into existence. We fill our lives with objects which were once upon a time mere blocks of material. But this is about to or at least, the way we do it

\section{ADDITIVE MANUFACTURING} 3D printing is on the rise. As the price of $3 \mathrm{D}$ printing has fallen, the previously expensive design tool has now gaine a niche hobby 3D pition for being .

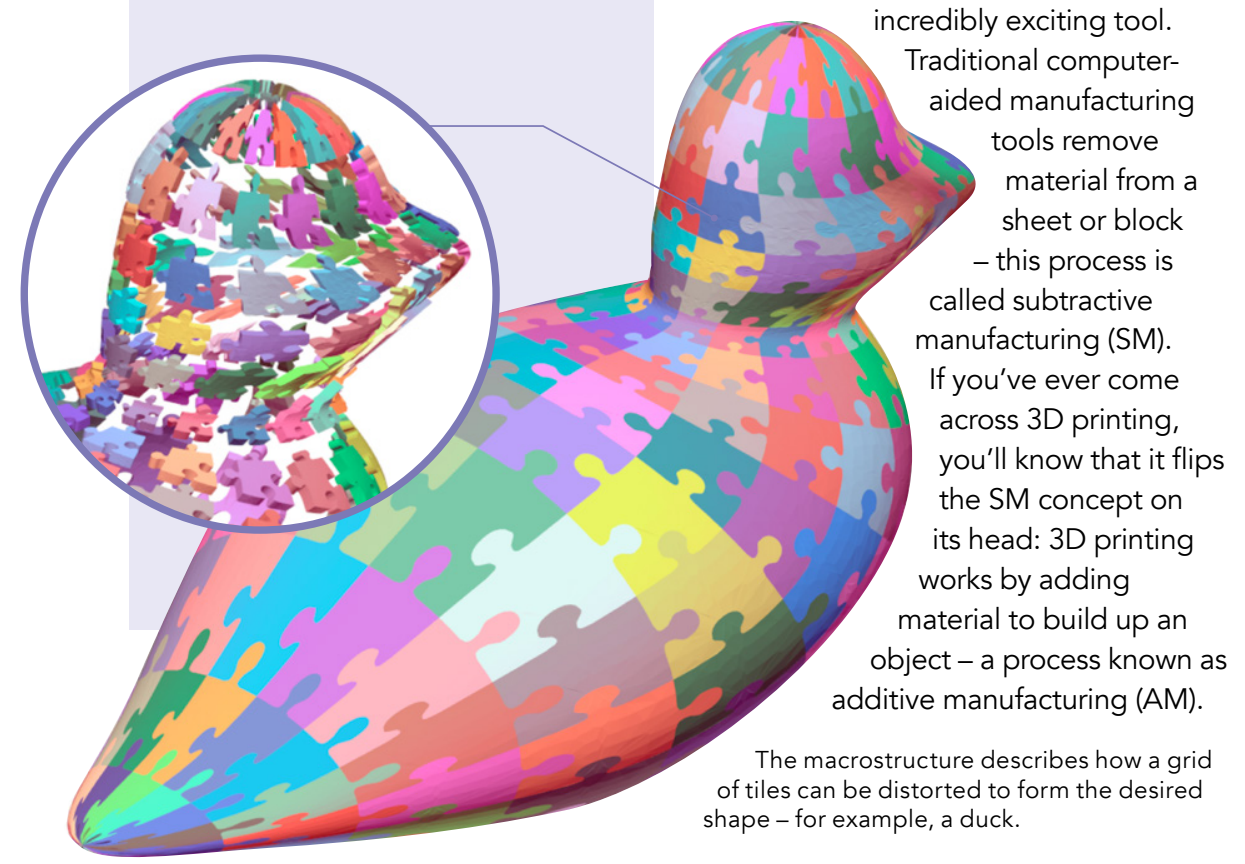
Hollow or even porous objects are werior strength-to-weight ratios, sis the possibility of is the possibilly of creating objects in composition throughout the object. With SM it's impossible to reach the inside of an object with a cutter head, and unfeasible to create objects with delicate microstructures with heavyhanded tools.

\section{D PRINTING: THE FUTURE?} The future is in 3D printers - or at least, it should be. But there's a problem. Because subtractive manufacturing has been the industry standard for so long, geometric modelling software is set up to reflect this. This software is designed to create virtual (computer) objects in the boundary representations, know In the industry as 'B-reps'. B-reps are or bounda Up unt now ro need to represent the interior of an object, because the cutter or drill head, water jet or laser beam responsible for shaping the object simply needs to know where to cut, and where not to cut. Everything within the boundary walls of an object is assumed solid and homogenous.

This is a major issue for additive manufacturing that supports heterogeneity and porosity. Never before were the (additive) manufacturing abilities ahead of geometric design software capabilities and a new set of software is needed to design, manufacture and analyse of manufacturing.

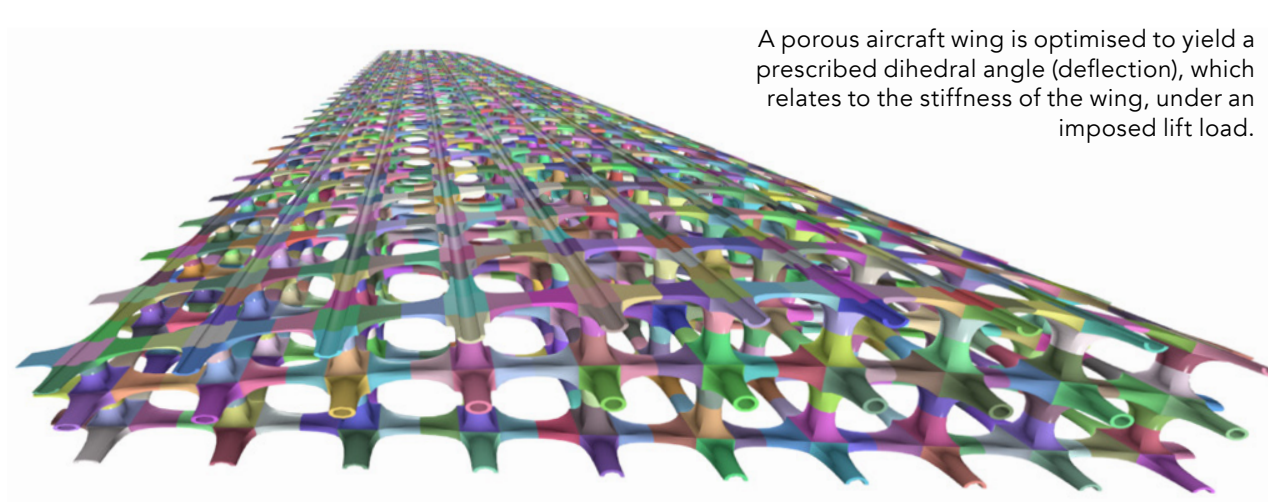

\section{VOXELS AND V-REPS}

There have been some attempts at making alternatives to B-reps. Howe up until now, none have emerged as particularly suitable. One of the most straightfoward approaches is to divide in technically work and is similar to the crel representations used in medical scans, but it also has serious limitations. Voxel representations are incompatible with B-reps. What's more, applications requiring accuracy on the same order as the modern SM methods may need terabytes for a single object.

Gershon Elber, Professor of Computer Science at Technion, Israel, has been working on the development of a different approach that is also B-rep compatible, employing the same mathematical functions. This approach which he calls '-reps', short-hand for volumetric representations, is a more for printing.

The development of $\mathrm{V}$-reps fruited first in a 2016 paper, which details a method for using mathematical functions to construct heterogeneous and/or porous objects. One premise is that the g designed can be constructed from two descriptions of its structure: the microstructure an

The microstructure is a 3D representation consisting of numerous tiles, whereas a 'tile' is a stracture which wil be repeated throughout the macrostructure. The microstructure tile is typically repeated manip-dimensional grid, which is then manipulated into the sha
The macrostructure essentially describes how a grid of tiles can be distorted to conform to the desired object's shape. This shape can be as simple as a cuboid grid which appears to have much more complex, requiring multiple Boolean operations (mathematical operations like AND, OR, NOT that combine or exclude simple objects resulting in a more complex object) to achieve the desired shape. Prof Elber has various objects represented in $h$ papers, but one which consisten shows up is a duck - a testbed complicated to programme.

Objects are already represented volumetrically, removing the need to

convert models between different representations, so objects can be very quickly iteratively optimised.



Each wing is made out
to different extents. (nside, Elber's more recent work wat that manages and optimises properies topological and materia properties of individual tiles. Within model, tiles can be designed with variety of shapes and form, and to neighbouring tiles on one face or multiple, smaller versions on another
face, etc. Further, the tiles themselves may form out of smaller tiles, recursively which are branched, hierarchical structures. The resulting collection of thes can, ike previously, be transformed (n) approach to powerful. Not only is it relatively light pomputation load and memory use, analysis. To analyse the properties of an object, designers currently convert the object's B-rep into a mesh which describes its volume. The mesh representation allows designers to model different parameters across the object - for example, heat or stress and 


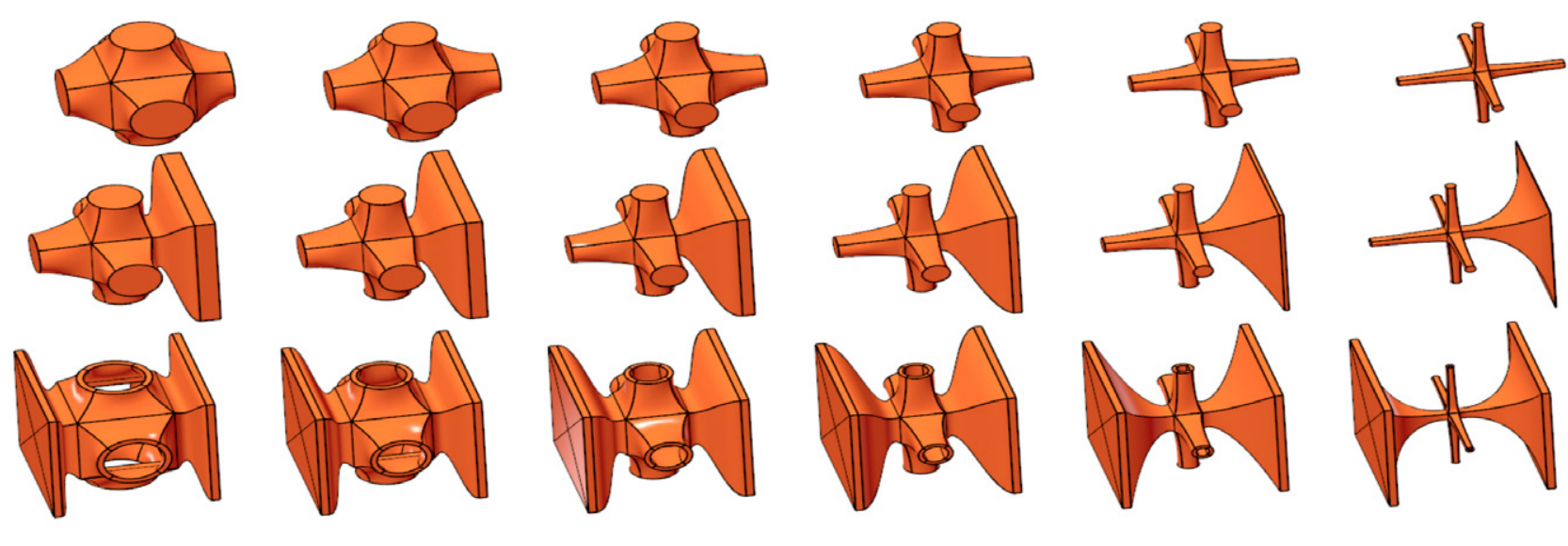

$V$-reps are capable of aiding in the design of a variety of objects, including heat sinks, heat exchangers, solid heterogenous rocket fuel, aeroplan
wings and plastic extrusion nozzles. Shown are samples from continuous families of parametric tiles

strain. In the automotive, aerospace and ship-bulling industries - some of the biggest users of computer-aided is estimated to consume $80 \%$ of the overall design and analysis process.

What makes Elber's design method a big hitter is that objects are already represented volumetrically, and hence analysis-compatible, removing the need to convert models, so objects between different representations can be very quickly iteratively optimised.

\section{DESIGNING A POROUS}

AIRCRAFT WING

The fact that objects in Elber's V-rep design process are tiled makes the

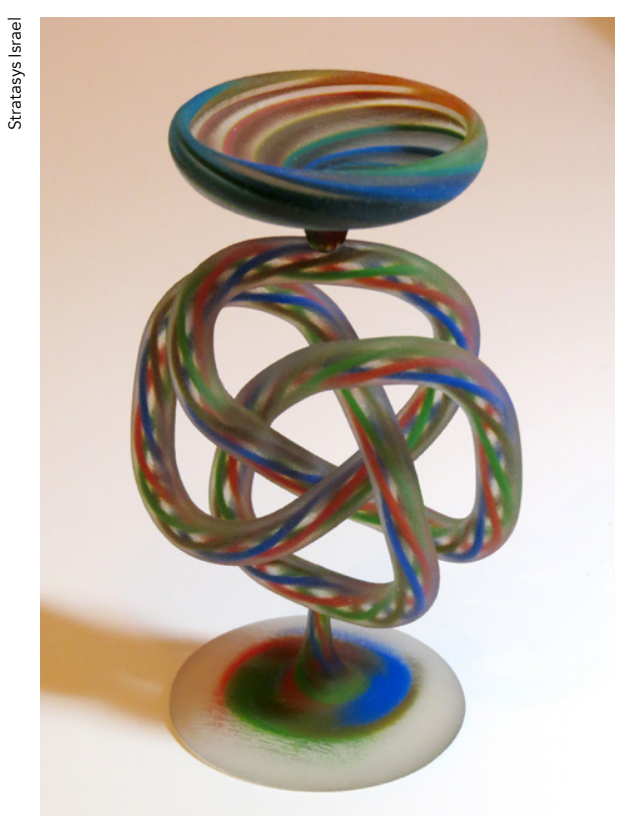

A 3D printed V-rep model
made with heterogeneous materials.
The optimisation software could create completely novel objects. Maybe one day, you'll own one.

Elber demonstrates this with several pre-mixing materials in a mixing designs for porous tiled aircraft wings, chamber. Needless to say, subtractive

dihedral angle (deflection), which relates close to matching this level of local to the stiffness of the wing, under an imposed lift load.

Each wing is made of thousands of tiles, each of which can be altered to contribute to the overall optimisation of an object, by varying their thicknesses, and hollowing out their insides to different extents. Elber's designs incorporate gradual changes in tile thickness along four wing designs. These examples are showing that optimising the distribution of thicknesses optimally light and stiff wing designs.

\section{HETEROGENEOUS COMPOSITION} Elber is looking towards the creation of software that will satisfy the present and future foreseen needs and abilities the ability to specify throughout the object what the material composition will be. This specification can come from the end user, but more realistically from analysis and optimization tools. 3D printer manufacturers are competing to create the hardware which could suppo heterogeneous matentals, while shis technology is still in its infancy. Current approaches generally either involve which are optimised to yield a prescribed manufacturing techniques can't com of AM. Built into the V-rep software is control over materia.

\section{OPTIMISATION OF V-REPS}

Elber's most recent work describes furtherimprovements to the optimisation process, utilising an automated optimiser prom schemes. In the case of the wings, this over microstructure geometry like wall thickness and pipe diameter, but possibly also over the material compostion

He demonstrates that the technology ing in the design of a rocket fuel, aeroplane wings and plastic extrusion nozzles, all of which were optimised at a microstructure level.

Elber suggests that in the near future, optimisation functions could be given control over other parameters, allowing them to embed nano-tiles within the tiles, etc., and choose between different types of tiles in each section of the software could create completely novel optimiser programme was given contro variety of objects, including heat sinks, heat exchangers, solid heterogenous current micro-tiles, pico-tiles within nanogrid and even control the deformation function, meaning that the optimisation

\section{Dr Gershon Elber}

W: gershon@cs.technion.ac.il T: +972 544717317

Research Objectives

Prof Elber explores the shift from subtractive to dditive manufacturing and the associated new $3 D$

\section{Detail}

Gershon Elber

Department Technion,

Haifa 32000 , Israel

Bio

Gershon Elber has been a Professor at the Computer Science Department Technion, Israel, for almost thirty years. His research interests span from computer-aided geometric designs to computer graphics. Prof Elber received the John Gregory Memorial Award in 2011, the Solid Modeling Association pioneers award in 2016, and the Bezier award in 2019.

Funding

Agency, USA

EC (European community, European Innovation council) ISF (Israel Science Foundation)

\section{Collaborators}

(Annalisa Buffa, Elaine Cohen, John Machchar Massimiliano Martinelli, Fady Massawi

Richard Riesenfeld, Giancarlo Sangalli, Boris van Sosin

\section{$\sqrt{7}$ Technion}

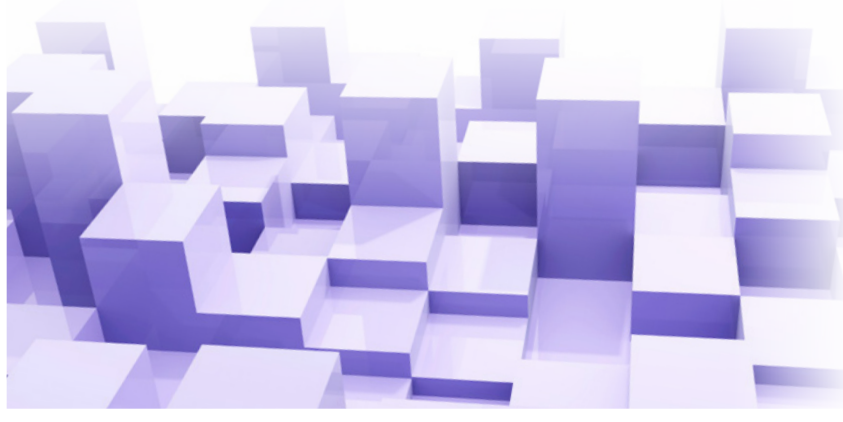

\section{References}

Massarwi, F. and Elber, G. (2016). A B-spline based framework for volumetric object modeling. ComputerAided Design, 78, 36-47.

Elber, G. (2016). Precise Construction of Micro-structures and Porous Geometry via Functional Composition. The Mathematical Methods for Curves and Surfaces (MMCS9) June 2016, Tonsberg, Norway, M. Floater et al. (Eds.): MMCS 2016, LNCS 10521, 108-125.

Ezair, B., Dikovsky, D. and Elber, G. (2017). Fabricating functionally graded material objects using trimmed trivaria volumetric representations. Proceedings of SMI.

Massarwi, F., Machchhar, J., Antolin, P. and Elber, G. (2018). Hierarchical, random and bifurcation tiling with heterogeneity in micro-structures construction via functional composition. Computer-Aided Design, 102 148-159.

Antolin, P., Buffa, A., Cohen, E., Dannenhoffer, J. F., Elber, G., Elgeti, S., et al. (2019). Optimizing micro-tiles in microstructures as a design paradigm. Computer-Aided Desig

\section{Personal Response}

In your papers you model objects using V-reps. and types of objects where $V$-reps and AM could be influential?

II Being able to fabricate heterogeneous or are typically coined, and more so porous FGM objects, brings a whole new ball to the game. Every field in which weight plays a critical role can benefit from that
new ball. Aerospace immediate comes to mind but so do wearable electronics that must be as light as possible, as well as medical implants. While we can see today the impactful benefit of porosity, artificial FGM objects virtually never existed before the AM era and the full benefit of FGM is yet to be revealed. II 